\title{
A Comparative Analysis of the Quadratus Lumborum Block Versus Femoral Nerve and Fascia Iliaca Blocks in Hip Arthroscopy
}

\author{
Ryan E. Blackwell, M.D., Michael Kushelev, M.D., John Norton, D.O., Robert Pettit, M.D., \\ and W. Kelton Vasileff, M.D.
}

\begin{abstract}
Purpose: To evaluate the effect of the single-shot quadratus lumborum (QL) block versus femoral nerve and fascia iliacus (F/FI) blocks performed preoperatively on perioperative opioid requirements, subjective pain scores, and time to discharge. Methods: Patients who underwent hip arthroscopy for femoroacetabular impingement and had a preoperative nerve block between January 2017 and August 2019 at our institution were identified. Patients were separated into 2 groups: those who either received a preoperative single-shot QL block or a preoperative single-shot F/FI block. All patients received general anesthesia. Intraoperative, postanesthesia care unit (PACU), and total morphine equivalents were analyzed using unpaired $t$ test. Secondary outcome measures including total time in PACU and block-related complications were recorded and analyzed as well. Results: One hundred one patients were retrospectively reviewed. Forty-three patients received preoperative QL blocks, and 58 patients received preoperative F/FI blocks. Demographics and operative characteristics were similar between the 2 groups. Patients receiving a QL block required significantly lower total morphine equivalents $(63.1$ vs 87.0, $P<.001)$. Patients receiving a QL block also had shorter PACU stays (116 vs 148 minutes, $P<.001)$ and lower subjective pain scores at the time of discharge $(3.27 \mathrm{vs} 4.98, P<.001)$ compared with the F/ FI block group. There were also significant decreases in the number of intraoperative opioids $(42.1 \mathrm{vs} 58.4, P<.001)$ and PACU opioids (20.7 vs $28.7, P=.03$ ) used when analyzed separately. Two patients in the femoral nerve block group had noted a fall postoperatively while the block was in effect. No patients in the QL block group had a block-related complication. Conclusions: Patients receiving a preoperative QL block for hip arthroscopy demonstrated lower total opioid requirements, shorter PACU stay, and lower pain scores at discharge than patients receiving preoperative F/FI blocks with no reported adverse events. Level of Evidence: Level III, retrospective comparative trial.
\end{abstract}

$\mathbf{H}$ ip arthroscopy has increased in prevalence as a method of treating patients with hip pathology as its indications, techniques, and instrumentation have expanded. ${ }^{1,2}$ The majority of these procedures are done

From the Department of Orthopaedics (R.E.B., R.P., W.K.V.), Department of Anesthesia (M.K., J.N.), and Sports Medicine Research Institute (W.K.V.), The Ohio State University Wexner Medical Center, Columbus, Ohio, U.S.A.

The authors report the following potential conflicts of interest or sources of funding: W.K.V. reports personal fees from Zimmer Biomet, outside the submitted work. Full ICMJE author disclosure forms are available for this article online, as supplementary material.

Received February 7, 2020; accepted August 18, 2020.

Address correspondence to William Kelton Vasileff, M.D., Department of Orthopaedics, The Ohio State University, Sports Medicine Research Institute, 2835 Fred Taylor Dr., Columbus, OH 43202.E-mail: William.Vasileff@ osumc.edu

(C) 2020 THE AUTHORS. Published by Elsevier Inc. on behalf of the Arthroscopy Association of North America. This is an open access article under the CC BY-NC-ND license (http://creativecommons.org/licenses/by-nc-nd/4.0/).

2666-061X/20178

https://doi.org/10.1016/j.asmr.2020.08.002 as an outpatient in hospitals or ambulatory surgery centers, given the minimally invasive techniques and limited soft-tissue trauma. Despite this, significant perioperative and early postoperative pain remains one of the most frequently cited challenges in hip arthroscopy. ${ }^{3}$ Adequate pain management following surgery is linked with increased patient satisfaction, earlier mobilization, decreased pain medication requirements, and overall improved outcomes. ${ }^{3}$ Despite this, pain following hip arthroscopy can be difficult to manage. The sensory innervation of the hip joint is complex, receiving innervation from the obturator, sciatic, femoral, superior gluteal nerves, and nerve-toquadratus femoris. ${ }^{3,4}$ Ensuring adequate pain control following arthroscopic surgeries involving the hip is a complex endeavor and often includes a combination of intravenous, enteral, regional, and local analgesia.

One of the more frequently used methods of pain control following orthopaedic surgery is the use of opioids. Orthopaedic surgeons are the third-highest 
prescribers of opioid prescriptions among physicians in the United States. In addition to the common side effects of opioids, opioid abuse has increased 3-fold in recent years. ${ }^{5}$ Of the more than 700,000 overdose deaths in the United States from 1999 to 2017, more than 400,000 of them involved an opioid medication. ${ }^{6}$ Alternative methods for postoperative analgesia and the effective formation of a multimodal pain regimen have been studied and frequently used in an attempt to decrease overall opioid prescriptions and improve patient outcomes. These can include over-the-counter analgesics, neuromodulators, and local anesthetics among others. Regional anesthesia with pre- or postoperative nerve blockade has been commonly used in orthopaedic surgeries and has become more important in the recent years of increased opioid abuse.

There exist multiple options for regional anesthesia in surgeries around the hip. The more common nerve blocks used in this population include femoral nerve, fascia iliaca, and lumbar plexus. Much of the literature regarding nerve blocks around the hip have surrounded open procedures such as arthroplasty and fracture treatment. ${ }^{7,8}$ Femoral nerve block has been shown to provide effective pain relief for patients following hip arthroscopy; however, a recent randomized control trial by Xing et al. showed an increased incidence of falls following femoral nerve block in these patients due to quadriceps inhibition., ${ }^{910}$ Generally, regional nerve blocks have proven effective in hip arthroscopy and are commonly employed as part of a multimodal pain control plan..$^{9-13}$ As the prevalence of hip arthroscopy has increased in recent years, so has the search for the most effective and safest technique for postoperative analgesia in these patients.

Quadratus lumborum (QL) blocks can be described as an injection into the thoracolumbar fascia surrounding the QL muscle, with diffusion of local anesthetics into the paravertebral space as cephalad as T6 and caudad as L3. ${ }^{14}$ Approaches for the QL block (lateral or QL1, posterior or QL2, and anterior or QL3) vary depending on the exact target for local anesthetic deposition in relation to the QL muscle, with no conclusive evidence to suggest the superiority of one approach to others for hip surgery. Although the best technique for performing a QL block is not well understood, QL blocks have been validated as an effective method of analgesia for patients with femoral neck fractures and following total hip arthroplasty. ${ }^{7,15}$ They have been shown to decrease intraoperative opioid use and reduce overall length of stay. ${ }^{7}$ The greatest benefit of the QL2 block remains the fact that it is a motor-sparing, effective technique for analgesia around the hip. Although the exact mechanism of action for QL blocks is not well understood, it is thought the posterior QL2 is largely motor-sparing due to its limited penetration into the psoas muscle and the L3 and L4 nerve roots. ${ }^{13}$
Thus far, there is limited research on QL blocks used for hip arthroscopy. In the current literature, one study has shown QL blocks to be superior to a multimodal pain regimen alone for improving perioperative pain and reducing opioid consumption; however, a second cohort study found QL blocks to not be an effective analgesic technique in hip arthroscopy. ${ }^{13,16}$ No studies to date have directly compared the effectiveness of QL blocks versus the more common femoral nerve or fascia iliaca (F/FI) blocks in hip arthroscopy. The purpose of this study is to evaluate the effect of the single-shot QL block versus F/FI blocks performed preoperatively on perioperative opioid requirements, subjective pain scores, and time to discharge. Our hypothesis was that the QL block would provide superior analgesia compared with femoral nerve and fascia iliaca blocks, resulting in lower opioid intake and improved patientreported pain scores.

\section{Methods}

After we received institutional review board approval for this study (approved by the Biomedical Institutional Review Board of The Ohio State University), a retrospective series was created of all patients who had undergone hip arthroscopy for femoroacetabular impingement by a single surgeon at our institution between January 2017 and August 2019. Both groups included patients undergoing surgeries throughout the entire study period. We identified all patients who had received a preoperative nerve block and then separated that cohort into 2 groups who either received a preoperative single-shot QL block or a preoperative single-shot femoral nerve or fascia iliaca block. Patients were excluded if they received spinal anesthesia, a postoperative "rescue" nerve block, had incomplete chart data, or had an open procedure. Inclusion criteria included standard indications for hip arthroscopy (both bony and soft-tissue pathology), a preoperative nerve block, and complete chart data.

Charts were retrospectively reviewed for patients in both groups. Basic patient demographics, type of procedure, operative time, and time in the postanesthesia care unit (PACU) were recorded. The main outcome measures gathered were pain scores, number of opioids received, and time to discharge. Our institution uses the Defense and Veterans Pain Reporting Scale (DVPRS) for subjective pain scores and these were recorded both on arrival to PACU and just before discharge. The amount of opioids received intraoperatively and postoperatively in PACU were converted to oral morphine equivalents (OME) using a standardized and validated conversion. ${ }^{17}$ Time to discharge was measured from the time the patient arrived in the PACU to the time they were discharged home. Charts were reviewed to identify any early postoperative complications such as falls, infection, or return to the hospital for pain control. 
Following informed consent, posterior QL blocks were administered by fellowship-trained anesthesiologists in the preoperative area under moderate sedation or after initiation of general anesthesia in the operating room depending on anesthesiologist's preference. Block selection was done by a combination of discussion with the patient and anesthesiologist training as well as comfort level with the various block techniques. Using ultrasound guidance and an anterior-to-posterior needle trajectory, 20 to $40 \mathrm{~mL}$ of $0.2 \%$ to $0.5 \%$ ropivacaine was injected on the posterior aspect of the QL muscle as described by Blanco et al. ${ }^{18} \mathrm{~A}$ multimodal analgesic regimen included preoperative acetaminophen 650 to $975 \mathrm{mg}$ and gabapentin 300 to $600 \mathrm{mg}$ with variations due to weight-based dosing, In the operating room, all patients received a balanced general anesthetic with an endotracheal tube. Anesthesia was induced with propofol, low-dose narcotics to blunt sympathetic response of direct laryngoscopy, and rocuronium to achieve muscle relaxation. Maintenance of anesthesia was maintained with some combination of inhalational agents and/or propofol infusion. Patients received intraoperative narcotics per standard anesthetic practice as well as Decadron $8 \mathrm{mg}$ and Toradol $15 \mathrm{mg}$. Infiltration by the surgeon was accomplished with $15 \mathrm{mg}$ of Toradol and ropivacaine $0.5 \%$ in the soft tissues and pericapsular area at the conclusion of the case. Standard PACU pain medication orders for all patients consisted of hydromorphone 0.2 to $0.5 \mathrm{mg}$ intravenously every 5 minutes and/or fentanyl $25 \mu \mathrm{g}$ every 5 minutes as needed; additionally, oxycodone 5 to $10 \mathrm{mg}$ orally as needed was administered to facilitate pain control in anticipation of discharge.

The $\chi^{2}$ test was used to compare categorical variables and 2-tailed unpaired $t$ test was used to compare continuous variables between the two groups. Statistical analysis was performed using Excel (Microsoft, Redmond, WA). Statistical significance was set at $P=.05$.

\section{Results}

A total of 101 patients were retrospectively reviewed. Forty-three patients received preoperative single-shot QL2 blocks, and 58 patients received preoperative femoral nerve or fascia iliaca blocks. Two patients were excluded before data collection from the cohort. One was excluded for receiving spinal anesthesia by patient request in addition to a QL block and the second due to the anesthesiologist not being confident of the accuracy of the block location due to significant patient motion during the procedure. The QL group consisted of 16 male $(37.2 \%)$ and 27 female $(62.8 \%)$ patients. The F/FI group consisted of 19 male $(32.8 \%)$ and 39 female $(67.2 \%)$ patients. There were no significant differences between the QL block and F/FI block groups in regard to average patient age $(34.0 \pm 11.3$ vs. $37.6 \pm 12.7$ years, $P=.14)$ or average body mass index $(26.8 \pm 6.3$ vs $28.9 \pm 5.6, P=.09$ ). Patients in the QL block group did have a shorter average operative time (1 18.5 \pm 25.9 minutes vs $135.7 \pm 30.1$ minutes, $P=.003$ ) (Table 1 ). There were no significant differences between the 2 groups in the proportion of patients undergoing a procedure involving bone $(88.4 \%$ vs $93.1 \%, P=.40)$ (Table 2).

Patients who received a QL block had significantly lower DVPRS scores at the time of discharge $(3.27 \pm 2.32$ vs $4.98 \pm 2.2, P<.001)$. Patients who received a QL block also received significantly fewer opioid medications intraoperatively $(42.1 \pm 18.3$ vs $58.4 \pm 20.4$ OME, $P<.001)$ and in the PACU $(20.7 \pm$ 16.7 vs $28.7 \pm 19.3$ OME, $P=.03$ ). Average time to discharge was significantly shorter in the QL block group as well $(116 \pm 26.9$ minutes vs $148 \pm 52.2 \mathrm{mi}-$ nutes, $P<.001$ ) (Table 3, Fig 1). Two patients in the femoral nerve block group had a fall noted on the day of surgery, with no documented postoperative complications in the QL block group.

In subgroup analysis, patients receiving QL block required an average of $63.10 \mathrm{OME}$ in the perioperative period. This is significantly fewer than those required by patients in the femoral nerve (81.64 \pm 29.8 , $P=.003)$ or the fascia iliaca $(96.55 \pm 26.8, P<.001)$ groups. There was not a significant difference in opioid requirements between the femoral nerve and fascia iliaca groups when compared directly $(P=.062)$.

\section{Discussion}

The results of the current study demonstrate that in our cohort, the QL block was more effective than femoral nerve and fascia iliaca blocks at reducing perioperative opioid consumption, improving subjective pain scores, and decreasing time to discharge following

Table 1. Demographic and Procedure Data

\begin{tabular}{|c|c|c|c|}
\hline & Quadratus Lumborum Block $(\mathrm{n}=43)$ & Femoral Nerve/FI Block $(\mathrm{n}=58)$ & $P$ Value \\
\hline Average patient age, y & $34.0 \pm 11.3(18-54)$ & $37.6 \pm 12.7(18-67)$ & .14 \\
\hline Average BMI & $26.8 \pm 6.3(17.9-43.9)$ & $28.9 \pm 5.6(19.8-44.0)$ & .09 \\
\hline Average case length, min & $118.5 \pm 25.9(60-196)$ & $135.7 \pm 30.1(91-228)$ & .003 \\
\hline Procedures involving bone, \% & 88.4 & 93.1 & .40 \\
\hline
\end{tabular}

NOTE. Numbers in parenthesis denote the range (min-max).

BMI, body mass index; FI, fascia iliaca. 
Table 2. Procedure Details

\begin{tabular}{|c|c|c|c|}
\hline & QL2 $(n=43)$ & Femoral Nerve $(\mathrm{n}=37)$ & Fascia Iliaca $(\mathrm{n}=21)$ \\
\hline Labral repair, n (\%) & $37(86.0)$ & $37(100)$ & $17(80.9)$ \\
\hline Femoroplasty, n (\%) & $38(88.3)$ & $34(91.9)$ & $16(76.2)$ \\
\hline Loose body removal, n (\%) & $29(67.4)$ & $25(67.6)$ & $16(76.2)$ \\
\hline Labral reconstruction, n (\%) & $4(9.3)$ & $1(2.7)$ & $2(9.5)$ \\
\hline Iliopsoas lengthening/release, n (\%) & $1(2.3)$ & 0 & $1(4.8)$ \\
\hline Ligamentum teres debridement, n (\%) & $1(2.3)$ & 0 & 0 \\
\hline Troch bursectomy, n (\%) & $1(2.3)$ & $1(2.7)$ & 0 \\
\hline Synovectomy, n (\%) & $1(2.3)$ & 0 & 0 \\
\hline Microfracture, n (\%) & 0 & $2(5.4)$ & 0 \\
\hline
\end{tabular}

NOTE. Columns denote the number of procedures of each type performed (n) followed by (\%). Multiple procedures were performed during each arthroscopy.

hip arthroscopy. The QL block group also exhibited fewer documented postoperative falls than the femoral nerve block group, which is hypothesized to be due to reduced muscle inhibition compared with femoral nerve blocks from decreased spread to the psoas muscle and lower lumbar nerve roots. ${ }^{13}$

Regional anesthesia has been commonly used for procedures around the hip, most frequently in hip fracture surgery and arthroplasty. In an effort to determine the most effective method of analgesia, studies have begun to investigate the effectiveness of the QL block for these procedures. A recent comparative study showed that hospital length of stay following total hip arthroplasty was found to be significantly decreased in patients receiving QL block compared with no block. ${ }^{7}$ Kukreja et al. ${ }^{8,19}$ found in 2 separate studies that both the anterior and posterior QL block are effective at decreasing opioid requirements following hip arthroplasty in the first 48 hours postoperatively. A case report of the QL block used for 2 cases of femoral neck fracture and revision hip arthroplasty found it provided significant pain control without hip flexor or quadriceps weakness. ${ }^{20}$ The results of these preliminary studies provide a promising outlook for research into the QL block for hip arthroscopy.

When evaluating hip arthroscopy, peripheral nerve blocks have been proven to be an effective method of analgesia with a relatively low complication rate. ${ }^{3,9-13}$
Dold et al. ${ }^{21}$ have reported that femoral nerve blocks were found to be more effective than general anesthesia alone with standard pain medications at reducing pain levels 60 minutes postoperatively. Similar findings have been seen with QL blocks for hip arthroscopy, which were shown to be superior to a multimodal pain regimen alone; however, procedures in this study were relatively short with very few labral repairs and femoroplasties performed, which may not be representative of the most common hip arthroscopy procedures performed. ${ }^{13}$ In contrast, a recent study of 15 patients receiving a QL block versus 54 patients in the control group by Kinjo et al. ${ }^{16}$ reported that, when compared with no regional block, QL blocks were not an effective analgesic technique for hip arthroscopy for femoroacetabular impingement. They acknowledge their relatively small sample size in the experimental group as a limitation and speculate that subtle differences in block location could lead to variable anesthetic spread. Also, every patient in both of their groups received an intra-articular ropivacaine injection at the case conclusion, which could affect early postoperative pain. No studies have directly compared the utility of the QL block versus the femoral nerve or fascia iliaca blocks in hip arthroscopy. In the only other study directly comparing QL block with femoral nerve block for surgery around the hip, Parras and Blanco ${ }^{15}$ found that patients in the QL block had lower perioperative opioid requirements and visual analog scale scores in the first

Table 3. Results

\begin{tabular}{lccc}
\hline & Quadratus Lumborum Block $(\mathrm{n}=43)$ & Femoral Nerve/FI Block $(\mathrm{n}=58)$ & $P$ Value \\
\hline Intraoperative opioids (OME) & $42.1 \pm 18.3(15-100)$ & $58.4 \pm 20.3(18-130)$ & $<.001$ \\
PACU opioids (OME) & $20.7 \pm 16.7(0-65)$ & $28.7 \pm 19.2(0-75)$ & $87.0 \pm 29.7(26-165)$ \\
Total opioids (OME) & $63.1 \pm 24.0(20-123)$ & $148 \pm 52.2(58-354)$ & $<.001$ \\
Time in PACU (min) & $116 \pm 26.9(80-197)$ & $4.98 \pm 2.20(0-8)$ & $<.001$ \\
DVPRS score at discharge & $3.27 \pm 2.32(0-7)$ & $<.001$ \\
\hline
\end{tabular}

NOTE. Denoted as mean \pm standard deviation (range).

DVPRS, Defense and Veterans Pain Reporting Scale; FI, fascia iliaca; OME, oral morphine equivalents; PACU, postanesthesia care unit. 
Fig 1. The graph shows the mean OME consumed by patients in the intraoperative $(P<.001)$ and postoperative $(P=.03)$ period, as well as the entire perioperative course $(P<.001)$. (OME, oral morphine equivalents.)

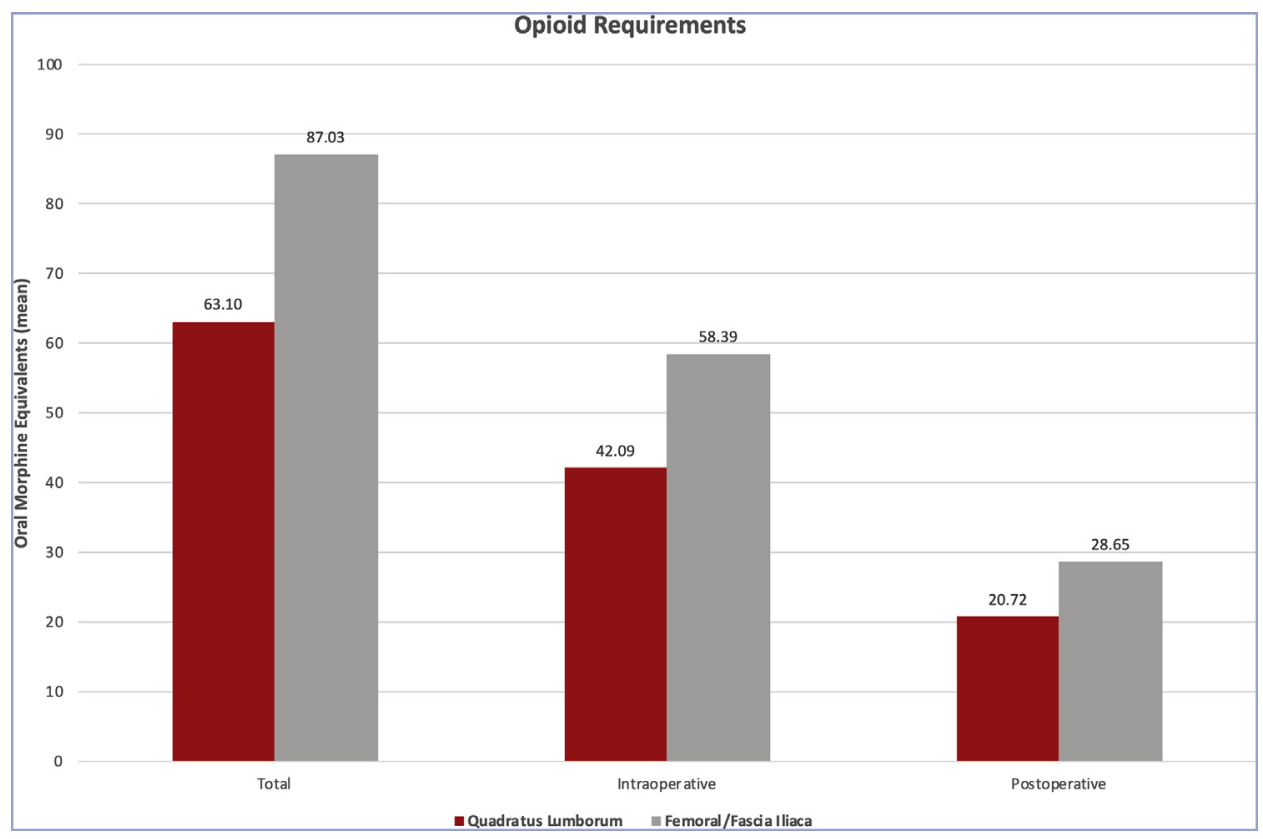

24 hours after hip hemiarthroplasty for femoral neck fracture.

More recent literature has shown that fascia iliaca blocks may not be an effective method of analgesia in hip arthroscopy for femoroacetabular impingement. ${ }^{22-25}$ We believe that by performing a subgroup analysis separating femoral nerve and fascia iliaca block patients and comparing each with the QL block group, this shows that our results are not skewed by the use of fascia iliaca blocks in some patients. The optimal regional anesthesia technique for hip arthroscopy has not yet been identified, likely due to the complex sensory innervation of the hip joint and limited available evidence. Given its wide anesthetic spread and predominantly sensory inhibition, the QL block theoretically has promise to be one of the most effective regional nerve blocks for hip arthroscopy.

Complications associated with femoral nerve and fascia iliaca blocks are well documented. Quadriceps inhibition leading to falls and prolonged sensory deficits are 2 of the most frequently cited block-related complications associated with femoral nerve and fascia iliaca block. In fact, in femoral nerve blocks rates of postoperative falls can reach $22 \%$. $^{9}$ Potter et al. ${ }^{26}$ found that cutaneous numbness after fascia iliaca block persisted through the first clinical follow-up visit in $26 \%$ of patients. Despite the relatively positive outcomes, QL blocks are not without risk. Ueshima and Hiroshi ${ }^{27}$ found that in their series of all techniques of QL blocks, up to $19 \%$ of patients had some level of quadriceps weakness after a posterior QL block, although the mechanism is unclear and time course of muscle weakness was not stated. Similar weakness was seen in one patient in a case report after QL block for gynecologic laparoscopy leading to unplanned admission. ${ }^{28}$ Despite these few reports, most sources describe QL blocks as a motor-sparing method of regional analgesia, and there was no documented lowerextremity weakness in any patients in our study. It is important to work toward identifying the most effective means of analgesia while minimizing what could be catastrophic complications.

\section{Limitations}

This study has several limitations. As a retrospective design rather than a prospective trial, there is some variability in concentration and amount of medication administered for individual blocks as well as likely subtle variations in the technique of block placement between different providers. However, all anesthesiologists at our institution placing QL blocks do so via the technique described by Blanco et al. ${ }^{18}$ In addition, as some patients received the preoperative block after induction of general anesthesia, not all blocks could be confirmed effective before the procedure. Further prospective trials with standardized block timing would be useful to assess for preoperative response to the block procedure.

As the study is not randomized, patient selection into the QL or femoral/FI groups could have potentially been influenced by a selection bias, either by patient preference or anesthesiologist preference. Given the fact there is not a control group (no block) included in the study, there are not data available for baseline opioid use, pain, or fall rates. With the design of the study, the data were only analyzed for the 
intraoperative and immediately postoperative period, and therefore factors such as pain or medication use after discharge as well as any late block-related complications cannot be accounted for. In addition, the group demographics were not entirely identical, with the QL block group having a lower average body mass index and a shorter average operative time. The operative time difference is likely due to a small number of relatively short cases (60 and 79 minutes) in the QL block group, although neither patient had unusually low opioid consumption (65 and 67.5 OME total).

When we compared the subjective pain scores, the minimum clinically important difference has not yet been established for the DVPRS score, and thus the clinical significance of the lower pain scores in the QL block group is unclear. Finally, specific information such as preoperative narcotic use and patient-reported outcomes were not gathered in this analysis.

\section{Conclusions}

Patients receiving a preoperative QL block for hip arthroscopy demonstrated lower total opioid requirements, shorter PACU stay, and lower pain scores at discharge than patients receiving preoperative F/FI blocks with no reported adverse events.

\section{References}

1. Truntzer JN, Shapiro LM, Hoppe DJ, Abrams GD, Safran MR. Hip arthroscopy in the United States: An update following coding changes in 2011. J hip Preserv Surg 2017;4:250-257.

2. Stone AV, Howse EA, Mannava S, Miller BA, Botros D, Stubbs AJ. Basic hip arthroscopy: Diagnostic hip arthroscopy. Arthrosc Tech 2017;6:e699-e704.

3. Shin JJ, Mccrum CL, Mauro CS, Vyas D. Pain management after hip arthroscopy: Systematic review of randomized controlled trials and cohort studies. Am J Sports Med 2017;46:3288-3298.

4. Birnbaum K, Prescher A, Heßler S, Heller KD. The sensory innervation of the hip joint-An anatomical study. Surg Radiol Anat 1997;19:371-375.

5. Morris BJ, Mir HR. The Opioid Epidemic. J Am Acad Orthop Surg 2015:23:267-271.

6. Scholl L, Seth P, Kariisa M, Wilson N, Baldwin G. Drug and opioid-involved overdose deaths - nited States, 2013-2017. MMWR Morb Mortal Wkly Rep 2018;67: 1419-1427.

7. Green MS, Hoffman CR, Iqbal U, Ives OO, Hurd B. Transmuscular quadratus lumborum block reduces length of stay in patients receiving total hip arthroplasty. Anesth Pain Med 2018;8:80233.

8. Kukreja P, Macbeth L, Sturdivant A, et al. Anterior quadratus lumborum block analgesia for total hip arthroplasty: A randomized, controlled study [published online October 25, 2019]. Reg Anesth Pain Med, https:// doi.org/10.1136/rapm-2019-100804.
9. Xing JG, Abdallah FW, Brull R, et al. Preoperative femoral nerve block for hip arthroscopy: A randomized, triplemasked controlled trial. Am J Sports Med 2015;43: 2680-2687.

10. Ward JP, Albert DB, Altman R, Goldstein RY, Cuff G, Youm T. Are femoral nerve blocks effective for early postoperative pain management after hip arthroscopy? Arthrosc J Arthrosc Relat Surg 2012;28:1064-1069.

11. Steinhaus ME, Rosneck J, Ahmad CS, Lynch TS. Outcomes after peripheral nerve block in hip arthroscopy. Am J Orthop (Belle Mead NJ) 2018;47(6).

12. Kay J, De Sa D, Memon M, Simunovic N, Paul J, Ayeni OR. Examining the role of perioperative nerve blocks in hip arthroscopy: A systematic review. Arthroscopy 2016;32:704-715.el.

13. McCrum CL, Ben-David B, Shin JJ, Wright VJ. Quadratus lumborum block provides improved immediate postoperative analgesia and decreased opioid use compared with a multimodal pain regimen following hip arthroscopy. J Hip Preserv Surg 2018;5:233.

14. Elsharkawy H, El-Boghdadly K, Barrington M. Quadratus lumborum block: Anatomical concepts, mechanisms, and techniques. Anesthesiology 2019;130:322-335.

15. Parras T, Blanco R. Randomised trial comparing the transversus abdominis plane block posterior approach or quadratus lumborum block type I with femoral block for postoperative analgesia in femoral neck fracture, both ultrasound-guided. Rev Esp Anestesiol Reanim 2016;63: $141-148$.

16. Kinjo S, Kolodzie K, Dong K, Zhang AL. The effects of transmuscular quadratus lumborum blocks on postoperative pain in arthroscopic hip surgery: A cohort analysis. J Anesth 2019;33:516-522.

17. Nielsen S, Degenhardt L, Hoban B, Gisev N. A synthesis of oral morphine equivalents (OME) for opioid utilisation studies. Pharmacoepidemiol Drug Saf 2016;25:733-737.

18. Blanco R, Ansari T, Girgis E. Quadratus lumborum block for postoperative pain after caesarean section: A randomised controlled trial. Eur J Anaesthesiol 2015;32: 812-818.

19. Kukreja P, MacBeth L, Potter W, et al. Posterior quadratus lumborum block for primary total hip arthroplasty analgesia: A comparative study. Einstein (Sao Paulo) 2019;17:eAO4905.

20. La Colla L, Ben-David B, Merman R. Quadratus lumborum block as an alternative to lumbar plexus block for hip surgery: A report of 2 cases. A A Case Rep 2017;8:4-6.

21. Dold AP, Murnaghan L, Xing J, Abdallah FW, Brull R, Whelan DB. Preoperative femoral nerve block in hip arthroscopic surgery: A retrospective review of 108 consecutive cases. Am J Sports Med 2014;42:144-149.

22. Glomset JL, Kim E, Tokish JM, et al. Reduction of postoperative hip arthroscopy pain with an ultrasound-guided fascia iliaca block: A prospective randomized controlled trial. Am J Sports Med 2020;48:682-688.

23. Huang MJ, Wages JJ, Henry AC, Epperson JM. Should preoperative fascia iliaca block be used for hip arthroscopic labral repair and femoroacetabular impingement treatment? A prospective single blinded randomized study. Arthroscopy 2020;36:1039-1044. 
24. Behrends M, Yap EN, Zhang AL, et al. Preoperative fascia iliaca block does not improve analgesia after arthroscopic hip surgery, but causes quadriceps muscles weakness: A randomized, double-blind trial. Anesthesiology 2018;129: 536-543.

25. Purcell RL, Nappo KE, Griffin DW, McCabe M, Anderson T, Kent M. Fascia iliaca blockade with the addition of liposomal bupivacaine vs plain bupivacaine for perioperative pain management following hip arthroscopy. Knee Surg Sport Traumatol Arthrosc 2018;26:2536-2541.
26. Potter MQ, Sun GS, Fraser JA, Beckmann JT, Swenson JD, Maak TG, et al. Psychological distress in hip arthroscopy patients affects postoperative pain control. Arthroscopy 2014;30:195-201.

27. Ueshima H, Hiroshi O. Incidence of lower-extremity muscle weakness after quadratus lumborum block. J Clin Anesth 2018;44:104.

28. Wikner M. Unexpected motor weakness following quadratus lumborum block for gynaecological laparoscopy. Anaesthesia 2017;72:230-232. 